\title{
Análisis de estrategias metodológicas docentes apoyadas en el uso de TIC para fomentar el Aprendizaje Cooperativo del alumnado universitario del Grado de Pedagogía
}

\author{
Eugenia FERNÁNDEZ MARTÍN
}

Datos de contacto:

Eugenia Fernández Martín. Universidad de Málaga. Campus de Teatinos. Facultad de Ciencias de la Educación. Bulevar Louis Pasteur, 25, 29010 Málaga (España).

eugeniaf@uma.es

\begin{abstract}
RESUMEN
Actualmente se está reconociendo cada vez más la importancia de las competencias auto-adquiridas del estudiante universitario en su proceso de construcción de aprendizajes, especialmente el aprendizaje cooperativo, para el correcto andamiaje del conocimiento académico y profesional, según el Espacio Europeo de Educación Superior (EEES). Por ello, el interés del presente estudio se centra en analizar las estrategias metodologías docentes más novedosas capaces de potenciar el aprendizaje cooperativo del alumnado.

En la presente investigación, se realizó un estudio de diseño mixto mediante correlatos estadísticos y evidencias de datos. Se seleccionó una muestra de 100 estudiantes del Grado de Pedagogía de la Facultad de Ciencias de la Educación de la Universidad de Málaga (España). Los instrumentos y estrategias de recogida de información fueron cuestionario, grupo focal, observación y análisis de documentos. Dicha recogida de información se realizó durante dos años académicos consecutivos, 2018-2019 y 2019-2020.

Los resultados de este estudio revelan que las estrategias docentes más eficaces para incentivar el aprendizaje cooperativo del alumnado, son las que se enfocan a propiciar la interacción con el grupo, la asunción de responsabilidades por parte del estudiantado, la implicación personal del mismo, y promover la participación tanto en el aula como, muy especialmente, en las plataformas virtuales universitarias, como se detalla a lo largo del presente escrito. Se concluye que estas novedosas estrategias metodológicas docentes, apoyadas en los actuales recursos tecnológicos, impulsan, fomentan y mejoran el aprendizaje cooperativo del alumnado universitario, lo que repercute directamente en la formación académica, profesional y personal del mismo.
\end{abstract}


PALABRAS CLAVE: Estrategia de enseñanza; TIC; Estudiante universitario; Enseñanza en equipo; Aprendizaje en línea; Trabajo en red.

\title{
Analysis of teaching strategies, supported in the use of ICT, to promote the Cooperative Learning of the university student of the Degree of Pedagogy
}

\begin{abstract}
Currently, it is really important the achievement of the students self-acquired competences in higher education, specially Cooperative Learning, for the suitable academic and professional development, according to criteria of the European Higher Education Area (EHEA). Therefore, the aim of the current study is focused on analyzing the most innovative teaching strategies are able to promote cooperative learning in Higher Education.

This study was designed with a mixed methods research, through statistical correlates and data evidence. The sample consisted of a total of 100 students from the Pedagogy Degree at the Faculty of Education Sciences of the University of Málaga (Spain). The instruments which were used for this research were questionnaire, focus group, observation and analysis of documents. The information was collected during two consecutive academic years, 20182019 and 2019-2020.

The results showed solid evidence of the teaching-learning methods that promote Cooperative Learning in higher education, are those that demands the use of teaching strategies as interaction, social skills, group reflection, heterogeneity, responsibilities, personal involvement, and promote participation both in university classrooms and, specially, in university virtual platforms, as detailed throughout this paper.

In conclusions, these innovate methodologies in university classrooms, supported on ICT, promote Cooperative Learning in Higher Education, which directly affects the academic, professional and personal student learning.
\end{abstract}

KEYWORDS: Teaching strategy; ICT; University student; Team teaching; Online learning; Networking. 


\section{Introducción}

En la actualidad, la formación inicial del alumnado universitario adquiere un papel fundamental, según el Espacio Europeo de Educación Superior (EEES), para formar un estudiantado competente en la consecución de aprendizajes y habilidades específicas para lograr la adquisición de importantes competencias profesionales (Korthagen, 2011, 2014; Gómez \& Ruíz, 2018; Martínez-Clares \& González-Morga, 2019), destacando entre ellas, el trabajo cooperativo (López Melero, 2018). Con esto, se posibilita al alumnado adquirir unos aprendizajes conjuntos y autónomos idóneos para conformar adecuadamente su rol profesional (Ibarra \& Rodríguez, 2011), ya que una de las habilidades que ha adquirido gran relevancia en el mercado laboral y la empleabilidad es el trabajo en equipo (Aguado, Arranz, Valera, \& Marin, 2011; Atxurra, Villardón, \& Calvete, 2015).

Por ello, el aprendizaje cooperativo es uno de los tipos de aprendizaje fundamentales para que el alumnado universitario llegue a una correcta y adecuada formación académica, profesional y personal (López Melero, 2014, 2018; Fernández \& Farzaneh, 2018; Korthagen, 2014), lo que conlleva la construcción social del conocimiento (López Melero, 2014; 2018), la reflexión e investigación grupal y profunda (Metlife et al., 2010; Korthagen, 2011), y la cooperación mediante el intercambio y la relación constante entre teoría y práctica (Fullan et al., 2015; Fernández Martín, 2009; Sepúlveda, Gallardo, Mayorga, \& Madrid, 2017).

Siguiendo esta línea, la metodología docente que se desarrolle en las aulas universitarias debe propiciar el aprendizaje cooperativo (López Melero, 2018; Biggs \& Tang, 2011; Cavanagh, 2011; Hammond, Bithell, \& Jones, 2010), no solo mediante el trabajo conjunto y autónomo (Zeichner, 2010; Ibarra et al., 2011), sino además mediante el dialógo (Well, 2001), la participación (García \& López, 2012), la comunicación (Fernández \& Farzaneh, 2018; Korthagen, 2011, 2014) y, en definitiva, la construcción del conocimiento a través de la interacción social (López Melero, 2014, 2018), llegando de esta menara a un aprendizaje, no solo conjunto, comunitario y compartido (Hargeaves \& Fullan, 2014), sino además, y en consecuencia, a un aprendizaje social (López Melero, 2014, 2018).

En este sentido, el uso de actuales recursos tecnológicos, puede proporcionarnos múltiples ventajas para tal fin, como herramientas didácticas de gran eficacia (De la Rosa, 2016; Moriña, Perera, \& Aguilar, 2014; Gómez \& Ruíz, 2018).

En el contexto universitario, hay a nuestra disposición múltiples y 
novedosos recursos digitales (Pinos Castillo, 2018; Suárez Lantarón, 2017; Valenzuela \& Hernández, 2017) capaces de facilitar y favorecer la comunicación, la relación, el diálogo y el contacto entre estudiante y profesorado, siendo aspectos fundamentales para un correcto proceso académico y educacional (Fullan et al., 2015; Korthagen, 2011, 2014; López Melero, 2014, 2018; Melief et al., 2010). Sumándose además, la inherente adaptación de las TIC a todas las peculiaridades y necesidades de las personas implicadas (De Carrillo \& Choquet, 2016; Faura, Martín, \& La Fuente, 2017), la acomodación espaciotemporal, y la adaptación al alumnado nativo digital (Cabero, Fernández, \& Barroso, 2016; De Carrillo \& Choquet, 2016).

En esta linea, existe el uso de plataformas virtuales de formación, específicamente en la Universidad de Málaga (UMA), existe el Campus Virtual (CV), basado en LMS/CMS Moodle, que es un sistema de gestión de cursos (CMS, Course Management System), un sistema de gestión de aprendizaje (LMS, learning management system) o entorno virtual de aprendizaje (EVA), que propicia la creación de un espacio educativo para grupos de formación universitaria (Aguaded \& Cabero, 2013; León de Mora, 2010).

Dentro del CV, existen distintas herramientas para potenciar en el alumnado habilidades específicas tan importantes como el mencionado aprendizaje cooperativo (Fernández, 2018; FernándezMartín \& Farzaneh, 2018; López Melero, 2018) y el aprendizaje conjunto (Hargeaves \& Fullan, 2014).

Para profundizar en cómo fomentar el trabajo cooperativo a través de las plataformas de formación universitaria, hay que atender a las distintas herramientas, ente ellas destacan:

- $\quad$ El foro de participación, en este espacio todas las personas implicadas en el proceso de enseñanza y aprendizaje pueden escribir y dejar un registro y seguimiento de sus ideas o interesas respecto a la asignatura, así como leer los comentarios del resto de participantes, entre ellos el profesorado, permitiendo el intercambio de ideas, cuestiones, comentarios, sugerencias, etc. Esto implica la resolución de dudas de manera colectiva, así como compartir conocimientos para la construcción de aprendizajes conjuntos mediante la cooperación y la participación (Fernández Martín, 2018; García \& López, 2012). Asimismo, implementa la tutorización colectiva, que permite al alumnado aprender de manera conjunta (Hargeaves \& Fullan, 2014).

- El envío de tareas con la opción de retroalimentación, es un elemento privilegiado para establecer un feedback continuo 
del trabajo grupal -o individual- que se va realizando entre alumnado y profesorado (Fernández-Martín \& Aranda, 2019). Permite un registro del proceso de aprendizaje y autoevaluación del estudiante, así como la evaluación continua. Aunque para ello, es imprescindible que el docente proporcione comentarios del profesor/a para cada tarea enviada, aportando correcciones, sugerencias, recomendaciones, o mejoras al trabajo cooperativo propuesto.

- El espacio de tutorización, aunque es creado normalmente para el seguimiento y orientación de las prácticas y Trabajos de Fin de Grado o de Fin de Master, supone una ventaja para el asesoramiento y seguimiento del alumnado en pequeños grupos, así como la selección del alumnado por parte del profesorado, de manera que se facilita y focaliza el seguimiento de los conocimientos y aprendizajes conjuntos del equipo de trabajo.

- Videoconferencias, muy útiles en el apoyo al proceso de enseñanza y aprendizaje en el contexto universitario (Pinos Castillo, 2018), como el Seminario Virtual B dentro del CV, que se basa en el programa open source llamado "BigBlueButton BN", es una forma de comunicación donde se interacciona visualmente en un diálogo inmediato que se dirige exclusivamente a los participantes conectados. Asimismo, existe también la opción de Seminario Virtual C, que se basa en el programa Black Board Collaborate, y consiste igualmente en una sala virtual en la que alumnado $\mathrm{y}$ porofesorado pueden comunicarse mediante audio y vídeo. La diferencia con el anterior, es que permite su uso a más personas al mismo tiempo.

- Wiki, es un módulo de actividad que permite a los participantes crear, modificar o añadir su contenido, en este caso páginas web, de forma fácil y rápida. Es ideal para el trabajo en equipo, ya que un wiki puede ser colaborativo, donde todo el alumnado puede participar, aportar y modificar la actividad.

- Taller, permite que el alumnado envíe su trabajo, y que pueda ser recopilado, revisado y evaluado por pares por sus propios compañeros/as. Puede ser un recurso excelente siempre que el profesorado lo enfoque como trabajo cooperativo, ya que este módulo de actividad permite a los estudiantes evaluar -o mejorar- los envíos de sus compañeras y compañeros. 
- Chat dentro del CV, permite la correspondencia entre todos los implicados que quieran unirse, para resolver dudas de manera conjunta o llegar a un conocimiento grupal. Es recomendable que el profesorado lo habilite en un periodo de tiempo determinado y hacerlo saber a todo el alumnado. Es ideal para la interacción inmediata. Ahora bien, una vez que se abandona el espacio virtual, no permanece el registro de las aportaciones.

- Otros recursos TIC fuera de Moodle: Redes sociales, pueden ser una herramienta privilegiada en el contexto académico (Chávez, 2015; León de Mora, 2010; Marín \& Cabero, 2019; Rodríguez, López, \& Martín, 2017; Suárez Lantarón, 2017). Como la mensajería instantánea WhatsApp (Suárez Lantarón, 2017) o Skype, mediante la creación de grupos donde se facilitan las aportaciones, participación, comunicación y cooperación inmediata del grupo de trabajo creado. Asimismo, Instagram, Twitter, o incluso Facebook (Heredia, Romero, \& Amar, 2018) son recursos que pueden apoyar, y completar la docencia y el aprendizaje (Rodríguez, López, \& Martín, 2017; Ruíz, 2019; Marín \& Cabero, 2019; López, 2019; Odetti \& Valentiluz, 2017) mediante la correcta utilización de estos medios y la adecuada preparación del profesorado (Cejas, 2018).

El profesorado es obviamente un elemento clave que determinará la metodología docente que se lleve a cabo en el contexto educativo, lo que constituirá y establecerá los tipos de aprendizajes que se fomenten en el alumnado universitario (Pozo \& Bretones, 2015; Rodríguez, 2014; Gómez \& Ruíz, 2018). Por consiguiente, un tipo de estrategia docente donde el profesorado diseñe y propicie actividades cooperativas adecuadas y pertinentes mediante la innovación, optimización y aprovechamiento de los recursos y técnicas a nuestro alcance (Marin \& Cabero, 2019; Heredia, Romero, \& Amar, 2018, Ruíz, 2019; López, 2019), conducirá a una mayor calidad y mejora del trabajo conjunto del alumnado, y del aprendizaje cooperativo en sí mismo (Gillies \& Boyle, 2010).

Así pues, el interés de este estudio tiene como objeto analizar cuáles son las más actuales y adecuadas estrategias metodológicas docentes -incluidas las apoyadas en el uso de las tecnologias de la información y la comunicación (TIC)-, que tienen un efecto directo en el aprendizaje cooperativo del estudiante, ya que es uno de los factores clave en la formación del alumnado universitario (EEES).

Por ello, el objetivo general del presente estudio se centra en: Analizar, valorar y comprender qué metodología docente es la más 
adecuada para propiciar y fomentar el aprendizaje cooperativo del alumnado universitario apoyándose en el uso de las TIC, desde el punto de vista de los protagonistas.

De este objetivo principal, se derivan los siguientes objetivos específicos:

- Indagar y conocer qué tipo de herramientas digitales se usan en el ámbito universitario para favorecer el aprendizaje cooperativo del estudiante.

- Valorar, conocer y entender cómo y en qué medida el uso de actuales recursos virtuales facilita el aprendizaje cooperativo del estudiantado universitario.

- Analizar, indagar, comprender y valorar la repercusión del uso de esta metodología docente en la formación académica del alumnado universitario.

\section{Metodología}

En coherencia con los objetivos, diseño y naturaleza de esta investigación, se ha diseñado un estudio mixto, para así conocer, comprender y dar respuesta a los objetivos de este estudio, con una pretensión muy específica y en un contexto muy delimitado (Simons, 2011); así como describir, analizar y examinar el foco de estudio, aportando de esta manera datos complementarios y adicionales para una mayor fiabilidad y objetividad de la investigación (Núñez Moscoso, 2017).

\section{Participantes}

Se seleccionó una muestra intencional no clínica de $\mathrm{N}=100$ estudiantes universitarios del Grado de Pedagogía de la Facultad de Ciencias de la Educación de la Universidad de Málaga (España). Todo el alumnado que conforma la muestra se encontraba matriculado en el cuatro y último curso académico, y pertenecientes a dos años académicos consecutivos, 2018-2019 y 2019-2020 (tabla 1), donde el $14 \%$ fueron alumnos y el $86 \%$ alumnas.

Tabla 1

Distribución de la muestra por curso académico

\begin{tabular}{ccccc}
\hline $\begin{array}{c}\text { № de } \\
\text { estudiantes }\end{array}$ & Curso & Año académico & Cuatrimestre & Grado \\
\hline 54 & 4⿻ & $2018-2019$ & 20 & Pedagogía \\
\hline 46 & 4⿻ & $2019-2020$ & 10 & Pedagogía \\
\hline
\end{tabular}




\section{Instrumentos y estrategias de recogida de información}

Las estrategias e instrumentos de obtención de datos seleccionados para llevar a cabo el estudio, fueron mediante grupo focal de discusión, observación y análisis de documentos relevantes para el análisis de corte cualitativo. Así como, la aplicación de cuestionario específicamente adaptado, no estandarizado, para el análisis de corte cuantitativo.

\section{Cuestionario adaptado}

Aplicación de un cuestionario no estandarizado, adaptado mediante la elección de ítems de dos cuestionarios que miden específicamente el aprendizaje cooperativo:

- Students' Evaluations of Educational Quality (SEEQ) de Marsh (1982, 1987, 1991), para valorar el Aprendizaje Cooperativo como Metodología de Enseñanza Aprendizaje en la Universidad. Seleccionando ítems como Interacción con el grupo, Implicación, y Asignaciones / responsabilidades.

- Escala de Aplicación del Aprendizaje Cooperativo (CLAS) de Atxurra, Villardón, y Calvete (2015), para medir el grado de cooperación que promueve el docente, seleccionando ítems sobre Interacción, Habilidades sociales, Reflexión grupal y Heterogeneidad.

El cuestionario resultante es una escala dicotómica con dos opciones de respuesta, donde 1 es "no necesariamente", y 2 es "sí, siempre sin lugar a dudas".

Esta adaptación del cuestionario fue presentada a 8 expertos en métodos de investigación y educación, concretamente 3 expertos y 5 expertas.

\section{Grupo focal de discusión}

Se usó la estrategia de grupo focal de discusión con cada grupo promocional del alumnado participante, ya que se pretende investigar los relatos y vivencias de los protagonistas de esta investigación sobre sus consideraciones personales y puntos de vista (Canales, 2006).

\section{Observación}

Se realizó la observación de cada una de las reuniones con el grupo focal para una mayor fiabilidad y validez al obtener información adicional y complementaria aportada por el lenguaje no verbal, las actitudes y los comportamientos. 


\section{Análisis de documentos e información recogida del CV}

Destacando la información recogida del foro de participación del $\mathrm{CV}$, donde se produjo la interacción entre participantes. Y el análisis de documentos e informes entregados al CV donde se incluyeron reflexiones y comentarios del alumnado sobre el foco de estudio.

\section{Procedimiento}

La recogida de información se realizó en la Facultad de Ciencias de la Educación de la Universidad de Málaga (España), durante el segundo cuatrimestre del año académico 2018-2019, y durante el primer cuatrimestre del 2019-2020 (tabla 1).

En ambos cursos se procedió de la misma manera, en primer lugar se realizó el estudio cualitativo, donde, tras la negociación de cuestiones éticas y previas durante el preacceso al campo de estudio, se desarrollaron las observaciones y reuniones con el grupo focal de discusión, de aproximadamente dos horas cada una de estas sesiones, y sobre la temática centrada en el objeto de estudio.

Una vez realizada la primera recogida de datos y tras localizar los indicadores (metodología docente) que el alumnado consideraba que más fomentaba su aprendizaje cooperativo, se realizó la investigación cuantitativa a través de cuestionario, concretamente un estudio descriptivo por cada estrategia docente considerada por el alumnado, a través de porcentajes, incidiendo en dos dimensiones: a) sin medios digitales, b) mediante el uso de las TIC.

\section{Análisis}

Los análisis de este estudio se realizaron durante y después de la recogida de datos, relazándose las técnicas de organización, codificación, y posteriormente de categorización. Tras ello, para aumentar la fiabilidad y validez del estudio cualitativo (Okuda \& Gómez-Restrepo, 2005) se aplicaron las estrategias específicas de triangulación, y finalmente de saturación.

Referente al análisis cuantitativo, tras localizar los indicadores perseguidos en el análisis anterior, se realizó un estudio descriptivo al respecto, a través del cuestionario adaptado (Marsh \& Roche, 1970; Atxurra, Villardón, \& Calvete, 2015), utilizando el software estadístico Statistical Package for the Social Sciences (SPSS) versión 25. E1 estudio descriptivo se llevo a cabo mediante el uso de porcentajes.

En este diseño mixto, ambos tipos de análisis no sólo tienen la ventaja de aportar información más complementaria y concluyente, 
sino que además es capaz de proporcionar un estudio más completo, fiable, válido, integro y exhaustivo (Johnson \& Onwuegbuzie, 2004; Campos Arenas, 2009; Núñez Moscoso, 2017), salvando de esta manera la posible parcialidad en la investigación.

\section{Resultados}

Los resultados más relevantes y significativos de esta investigación se exponen a continuación organizados en función de los objetivos del estudio.

\section{Estrategias metodológicas docentes para fomentar el trabajo cooperativo}

Los principales resultados de este estudio revelan que las metodologías docentes actuales más eficaces y adecuadas para incentivar y facilitar el aprendizaje cooperativo del alumnado universitario, según la perspectiva de sus protagonistas, son las que se enfocan a propiciar:

La Interacción con el grupo, la Implicación personal del alumnado, y las Asignaciones/responsabilidades del mismo, tanto en el aula como en las plataformas virtuales universitarias, como se evidencia en los siguientes datos.

Si el profesorado propicia la relación y la interacción con el grupo, es cuando podemos aprender cooperativamente. Pero en una clase tradicional, eso es imposible (Grupo focal de discusión, alumna de grado del pedagogía, 2019).

(...) Una metodología docente en la que, en el trabajo grupal, cada miembro del grupo tenga sus responsabilidades, hace que se adquiera una mayor implicación personal en la tarea y en lo que aprendemos en conjunto (Tarea reflexiva a cerca de la metodología docente que más fomenta el aprendizaje cooperativo. Alumna de grado del pedagogía, 2019).

Concretamente el $91 \%$ de los estudiantes participantes, consideran que la metodología docente encaminada a procurar una mayor Interacción con el grupo, es la que más les puede ayudar a fomentar el aprendizaje cooperativo dentro del aula (Figura 1).

Sin embargo, en las plataformas virtuales, el porcentaje es aún mayor en Interacción (98\%), seguido de la Implicación personal, y las Asignaciones/responsabilidades, con un 95\% para ambos (Figura 2). 


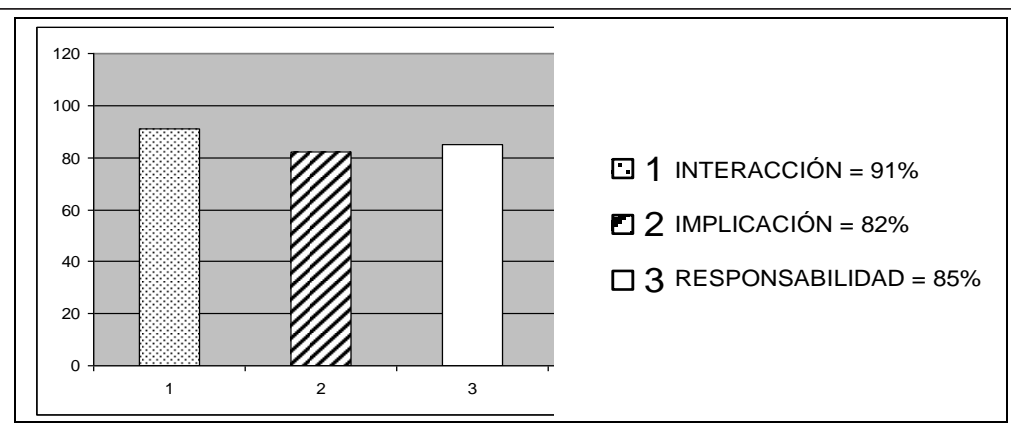

Figura 1. Estrategias metodológicas docentes que más fomentan el aprendizaje cooperativo en general.

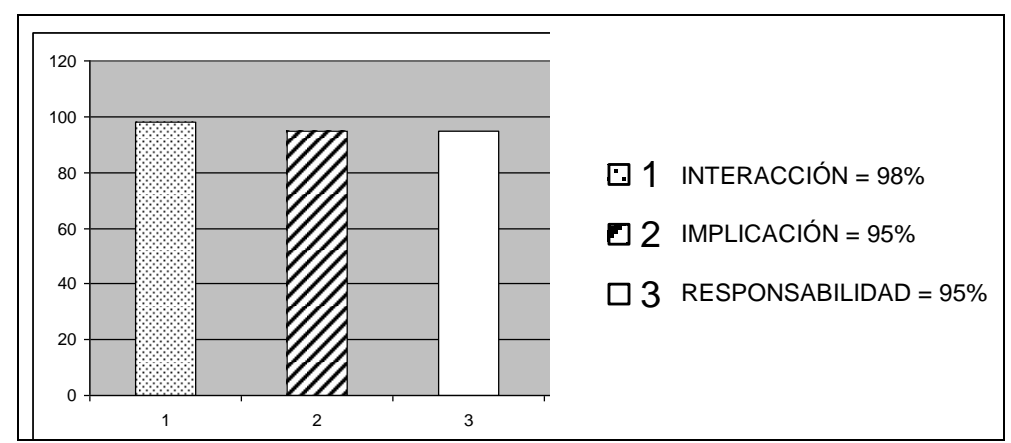

Figura 2. Estrategias metodológicas docentes que más fomentan el aprendizaje cooperativo mediante el uso de las TICs.

\section{TIC más utilizadas en el ámbito universitario para favorecer el aprendizaje cooperativo}

A continuación, se profundiza en los resultados del análisis de las herramientas o recursos virtuales de formación universitaria más usados tanto dentro como fuera de la plataforma Moodle.

\section{Foro}

El tipo de herramientas digitales que se usan en el ámbito universitario capaces de facilitar más el aprendizaje cooperativo del estudiante, se centran en el uso de plataformas de formación universitaria como el $\mathrm{CV}$, concretamente una de ellas es el foro de participación que favorece el aprendizaje conjunto y la cooperación, como se expone en las siguientes evidencias.

En el foro del CV se resuelven muchas dudas que preguntan los compañeros y compañeras, porque lo que no sabe uno, a lo mejor lo sabe otro. $Y$ nos ayudamos mucho entre todos porque a veces estamos en la misma situación... (Grupo focal de discusión, 
alumna de grado del pedagogía, 2019).

El foro de participación, me ha servido para guiarme, resolver dudas (...) y poder aprender de mis compañeros. (Tarea reflexiva, alumna de grado del pedagogia, 2019).

\section{Espacio para el envio de tareas}

El espacio para el envío de tareas, tiene la opción de registrar comentarios del profesorado y comentarios del alumnado acerca de la tarea grupal enviada, lo que favorece la retroalimentación del trabajo en equipo que se va elaborando y enviando de manera continua, contribuyendo a potenciar el avance, conocimiento y aprendizaje grupal.

Las observaciones que haga el docente sobre el trabajo grupal (...), es lo que más nos ayuda a aprender y a presentar un buen trabajo. (Grupo focal de discusión, alumna de grado del pedagogia, 2019).

\section{Espacio para la tutorización}

El espacio de tutorización, aunque normalmente es creado para el apoyo y orientación de los TFG y TFM, tiene la capacidad de incluir a pequeños grupos, y por tanto, concentrar el seguimiento a un equipo de trabajo concreto. Ahora bien, su uso debe tener como objeto el seguimiento del trabajo grupal cooperativo, y no para el seguimiento de trabajos individuales, según el punto de vista de los protagonistas del presente estudio.

Es más eficaz la orientación del profesorado si se enfoca a grupos concretos, ya que asi se centra en tu trabajo grupal y no en todos en general (Grupo focal de discusión, alumna de grado del pedagogia, 2019).

El espacio de tutorización de CV tiene sentido si se usa para crear conocimientos y aprendizajes comunes y grupales, no para mandar y enviar tareas individuales. (Reflexión, alumna de grado de pedagogia, 2019).

\section{Videoconferencias}

A tenor de las evidencias, dentro del CV, el Big Blue Button BN o Seminario Virtual B, permite la comunicación y visualización en directo del grupo clase. Aunque cada uno de los participantes tiene que estar conectado y disponer de los recursos apropiados para ello. Posibilita ampliamente la flexibilidad espacial, pero no temporal que es 
marcada por el profesorado.

Las videoconferencias (...) estaban genial, ya que se podia ver $y$ hablar con el profesor, escuchar a los demás compañeros (...). (Grupo focal de discusión, alumna de grado del pedagogía, 2019).

Si en ese momento exacto no te puedes conectar, o tienes un problema con el wifi... ¡Adiós! (Grupo focal de discusión, alumna de grado del pedagogía, 2019).

\section{Chat}

El Chat del CV tiene la ventaja de permitir la comunicación instantánea e inmediata entre todas las personas que se unan en ese momento, permite la interacción y construcción del conocimiento grupal, y facilita que dudas generales se resuelvan de forma conjunta, según la perspectiva de los participantes de este estudio.

Gracias al Chat de la asignatura, hemos podido aclarar $y$ resolver dudas que surgian en el momento, porque escribias alguna cuestión que surgía en tu grupo, y la profesora (o profesor) respondia directamente. (Reflexión de alumna de grado del pedagogía, 2019-2020).

\section{Otros recursos TIC fuera de la plataforma Moodle.}

Los resultados de este estudio muestran que las redes sociales son otros recursos TIC, fuera de la plataforma Moodle, que favorecen ampliamente el aprendizaje cooperativo y colaborativo ya que los grupos de trabajo pueden comunicarse, organizarse, ayudarse y acordar fácilmente. Especificamente, destaca el uso de mensajería instantánea como WhatsApp principalmente, o Skype. Asimismo, destaca la utilización de Google-drive para compartir, aportar, modificar o incluir contenido en los trabajos en equipo, entre otros programas, como se extrae de las evidencias siguientes.

A mi, el grupo de whatsApp con mis compañeros del Grado, es lo que me ha ayudado en la carrera, y con lo que más he aprendido de los demás. (Grupo focal de discusión, alumna de grado del pedagogía, 2019).

Es ideal usarlo para los trabajos grupales, porque estamos más en contacto y nos coordinamos mejor. (Grupo focal de discusión, alumna del Grado, 2019-2020).

(...) mediante WhatsApp consultamos dudas entre nosotros $y$ además acordamos la hora para ponernos a trabajar (...), en Drive elaboramos el trabajo, ya que podemos escribir a la vez y chatear 
(...), la visión y la escucha nos facilita la comunicación, por ello utilizamos Skype o Zoom (...) nos permiten compartir las pantallas para ver lo que cada uno está trabajando. (Tarea reflexiva sobre utilización didáctica de redes sociales, alumno del Grado, 20192020).

\section{Facilitación del aprendizaje cooperativo a través del uso de las plataformas virtuales universitarias}

El uso de actuales recursos virtuales, tanto dentro como fuera de la plataforma Moodle, facilita y promueve de manera determinante los aprendizajes cooperativos y conjuntos del estudiantado universitario en la medida en que se procure, por parte del profesorado, un verdadero trabajo cooperativo mediante la propuesta de elaboración de tareas, actividades o proyectos de investigación donde sea imprescindible que se ayuden y aprendan unos de otros durante la labor en equipo, a través de una correcta participación, interacción, reflexión grupal y heterogeneidad dentro del grupo, como se extrae de las siguientes evidencias.

Las plataformas virtuales pueden fomentar el aprendizaje cooperativo si el profesorado promueve la participación del alumnado en ellas, si no, solo sirven para obtener información, encontrar documentos, el temario (...) (Grupo focal de discusión, alumna de grado del pedagogia, 2019).

La comunicación, relación e interacción con el grupo, es lo que nos ayuda a aprender unos de otros (aprendizaje cooperativo), lo que se facilita mucho con el uso de las tecnologías virtuales, ya que ayudan a estar en contacto (...) (Tarea reflexiva, alumna de grado del pedagogía, 2019).

En esta línea, concretamente, el 95\% del alumnado participante considera que una metodologia docente que incide directamente en su aprendizaje cooperativo, es en la que el profesorado promueve la Interacción entre estudiantes, seguido de la Reflexión grupal (89\%) y la Participación y Heterogeneidad dentro del grupo, con un $85 \%$ y $81 \%$ respectivamente (Figura 3). Ahora bien, cuando se tata específicamente del uso de plataformas virtuales, es donde destacan estrategias metodológicas docentes que apuntan, en este caso, a la Participación en primer lugar, concretamente un 97\% del alumnado participante así lo considera, seguido de la Interacción (90\%), como factores fundamentales (Figura 4). 


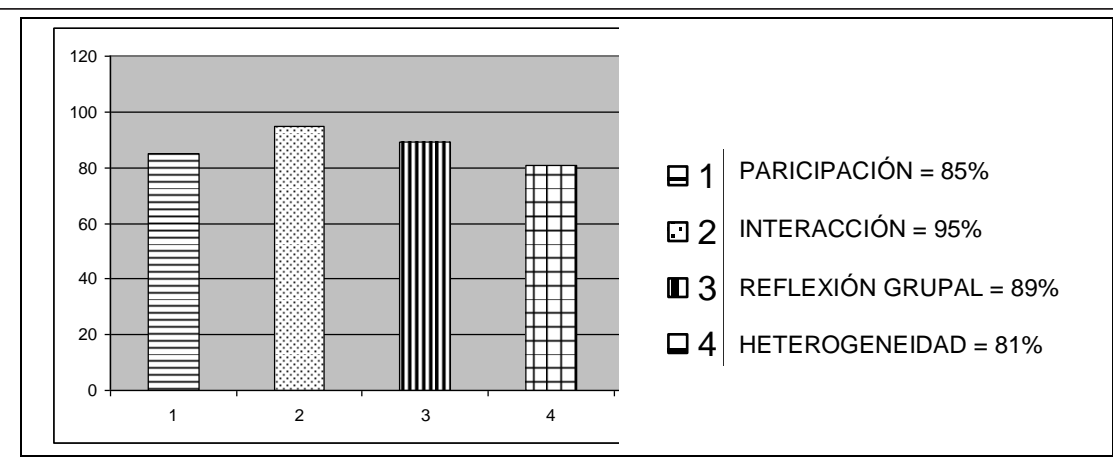

Figura 3. Estrategias metodológicas docentes que inciden directamente en el aprendizaje cooperativo del alumnado universitario.

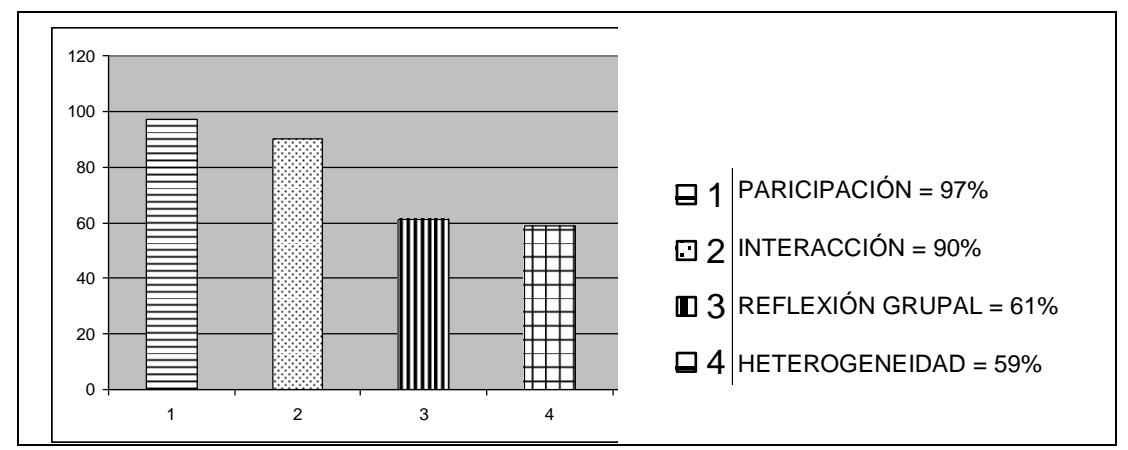

Figura 4. Estrategias metodológicas docentes a través de plataformas virtuales que inciden directamente en el aprendizaje cooperativo del alumnado universitario.

\section{Repercusiones directas en la formación del alumnado}

El uso de esta metodología docente, que ha demostrado fomentar el aprendizaje cooperativo, apoyada en los actuales recursos tecnológicos, repercute en la formación académica del alumnado universitario de manera significativa, en cuanto a que crece su autonomía personal, capacidad de trabajo en equipo y en conjunto, y sus habilidades sociales. Al mismo tiempo, con el uso de TIC, se fomentan habilidades como la responsabilidad, el empoderamiento, la participación e implicación personal, como se evidencia a continuación.

Este tipo de metodología en la que aprendemos mediante el trabajo cooperativo, me ha convertido en una persona más responsable, resuelta, participativa, y sociable.(...) Esta forma de trabajar, se facilita mucho con el uso de las tics, para poder comunicarte y contactar con tu grupo a pesar de no estar juntos fisicamente. (Tarea reflexiva, alumna de grado del pedagogía, 
2019).

Lo que destacaria que ha influido positivamente en mi formación son las habilidades profesionales de trabajo en equipo y de autonomía personal (Grupo focal de discusión, alumna de grado del pedagogía, 2019).

\section{Discusión y Conclusiones}

El aprendizaje cooperativo es un factor fundamental para la formación académica del estudiante universitario (EEES; Atxurra, Villardón, \& Calvete, 2015; López Melero, 2014, 2018; Fernández \& Farzaneh, 2018; Korthagen, 2014; Ibarra \& Rodriguez, 2011), pero su necesidad de implantación, y dificultad de promoción, está siendo una preocupación común hoy en día.

Se añade a esta dificultad, adaptar diferentes formas de enseñaza y aprendizaje al actual contexto educativo a través de la tecnología (Ruíz, 2019; Marín \& Cabero, 2019; Fernández-Martín \& Aranda, 2019; Fernández-Martín, 2018; Odetti \& Valentiluz, 2017), contemplando incluso el uso de redes sociales en el ámbito universitario (Fernández-Martín, 2020; Chávez, 2015; León de Mora, 2010; Heredia, Romero, \& Amar, 2018; Lopez, 2019; Rodríguez, López, \& Martín, 2017; Suárez Lantarón, 2017).

Como respuesta a dicha problemática, este estudio aporta evidencias en cuanto a que las estrategias metodológicas docentes que tienen un efecto directo en el fomento del trabajo cooperativo, y consecuentes aprendizajes conjuntos del alumnado universitario, son principalmente las destinadas a promover, por parte del profesorado, la interacción con el grupo de manera general; factor que se acusó más todavía, junto con la participación, si se implementaba el uso de TIC. Aunque también se evidenció la importancia de establecer responsabilidades dentro del equipo de trabajo, la implicación personal del alumnado, la reflexión grupal y la heterogeneidad dentro del grupo.

De ahí, la importancia del uso de las plataformas virtuales universitarias para aprovechar las herramientas que un sistema LMS como Moodle puede aportar durante el proceso de adquisición de aprendizajes del estudiantado, destacando, entre otras, el foro de participación, el espacio para el envío de tareas grupales con la opción de comentarios del profesor/a, y la creación de un espacio de tutorización para equipos de trabajo, como anteriormente se especificó. Asimismo, por su naturaleza sincrónica se recalca el Chat, $\mathrm{y}$ sobre todo las videoconferencias (Big Blue Button o Seminario 
Virtual B). Además, se evidencia la importancia y necesidad del uso de actuales recursos virtuales fuera de la plataforma Moodle, como las Redes Sociales, destacando principalmente el uso de la mensajería instantánea WhatsApp, u otras aplicaciones como Skype, Google-Drive o Zoom.

Ahora bien, para que estos recursos TIC, tanto dentro como fuera de Moodle, faciliten un verdadero aprendizaje cooperativo, y no la mera participación, es necesaria la habilidad docente de promover la elaboración de proyectos de investigación o trabajos grupales donde sea necesaria la ayuda y el apoyo común dentro del equipo de trabajo, así como el hecho de aprender unos/as de otros/as.

Este estudio aporta, además, que las mencionadas estrategias metodológicas capaces de impulsar el aprendizaje cooperativo, apoyadas en los actuales recursos tecnológicos comentados con anterioridad, repercuten directamente en la formación del alumnado universitario, en cuanto a que fomentan habilidades específicas ineludibles en un profesional de la educación-, como son la capacidad de trabajo en equipo, las habilidades sociales, la autonomía personal, la asunción de responsabilidades, y la capacitación de una apropiada utilización y aprovechamiento de recursos tecnológicos. Destrezas necesarias para formar a un alumnado competente y comprometido con la acción, con su futura profesión, y con la sociedad actual.

Se advierte la necesidad de que el profesorado universitario use estos recursos tecnológicos, no sólo como conducto de información y comunicación, sino también como aliado para fomentar el trabajo en equipo, la participación y el empoderamiento de su alumnado. Se propone la utilización didáctica y pedagógica, no solo de las herramientas anteriormente mencionadas disponibles en Moodle, sino además de las Redes Sociales como recurso complementario ideal para favorecer y facilitar el aprendizaje cooperativo de nuestro alumnado nativo digital, y adaptarnos a la actualidad social y educativa mediante nuevos entornos de enseñanza y aprendizaje.

\section{Referencias}

Aguaded, J. I., y Cabero, J. (2013). Tecnologías y medios para la educación en la e-sociedad. Madrid: Alianza.

Aguado, D., Arranz, V., Valera-Rubio, A., y Marín-Torres, S. (2011). Evaluación de un programa blended-learning para el desarrollo de la competencia trabajar en equipo. Psicothema, 23(3), 356361.

Atxurra, C., Villardón-Gallego, L., y Calvete, E. (2015). Diseño y 
validación de la Escala de Aplicación del Aprendizaje Cooperativo (CLAS). Revista de Psicodidáctica, 20(2), 339-357. DOI: 10.1387/RevPsicodidact.11917

Biggs, J., y Tang, C. (2011). Teaching for quality learning at University: What the student does. Maidenhead: McGraw-Hill. Open University Press.

Cabero Almenara, J., Fernández Batanero, J. M., y Barroso Osuna, J. (2016). Los alumnos del grado de Magisterio: TIC y discapacidad. Revista Electrónica de Investigación Educativa, 18(3), 107-120.

Campos Arenas, A. (2009). Métodos mixtos de investigación: Integración de la investigación cuantitativa y la investigación cualitativa. Bogotá: Magisterio.

Canales, M. (2006). El Grupo de Discusión y el Grupo Focal. En M. Canales (Ed.), Metodologías de investigación social (pp. 265-287). Santiago de Chile: LOM.

Cavanagh, M. (2011). Students' experiences of active engagement through cooperative learning activities in lectures. Active Learning in Higher Education, 12(1), 23-33. DOI: $10.1177 / 1469787410387724$

Cejas, R. (2018). La formación en TIC del profesorado y su transferencia a la función docente (Tesis doctoral). Barcelona: Universitat Autónoma de Barcelona.

Chávez, J.J. (2015). Uso de las redes educativas en la educación superior. Un caso específico. Revista Científica de Comunicación, 6(1), 82-96.

De la Rosa, L. (2016). La voz de las personas con discapacidad en la formación inicial. International Journal of Educational Research and Innovation (IJERI), 5, 160-169.

De Carrillo, C. P., y Choquet, C. (2016). Tutoria adaptativa mediante manipulación del contexto de aprendizaje en ambientes TIC. Cambios y Permanencias, 1, 41-65.

Faura, U., Martín, P. J., y La fuente, M. (2017). Un modelo conceptual para la realización del Trabajo Fin de Grado apoyado en el uso de las TICs. Revista de Educación a Distancia, 53, 7-31.

Fernández Martín, E. (2009). El valor educativo de los seminarios de 
trabajo del prácticum de la titulación de pedagogía de la Universidad de Málaga: Un estudio de caso. Málaga, España: RIUMA.

Fernández Martín, E. (2018). La innovación en la formación inicial del profesorado. Revista Innovamos, 9, 12-13.

Fernández-Martín, E. (2020). Estudio de las percepciones del alumnado universitario sobre el uso de redes sociales como herramienta didáctica en la formación inicial. En M. C. Pérez Fuentes (Ed.), Innovación Docente e Investigación en Educación. Madrid: Dykinson.

Fernández-Martín, E., y Aranda, L. (2019). Análisis del uso de herramientas digitales para facilitar el feedback como estrategia para la optimización del proceso de enseñanza aprendizaje. En M. C. Pérez Fuentes (Ed.), Innovación Docente e Investigación en Educación y Ciencias Sociales (pp. 627-634). Madrid: Dykinson.

Fernández-Martín, E., y Farzaneh, D. (2018). Voces Externas: Profesionales de la Educación que se Acercan al Proyecto Roma. Revista Interuniversitaria de Formación del Profesorado, 92(32.2), 151-162.

Fullan, M., Rincon-Gallardo, S., y Hargreaves, A. (2015). Professional capital as. accountability. Education Policy Analysis Archives, 23(15), 2-17.

García, M., y López, A. (2012). Explorando, desde una perspectiva inclusiva, el uso de las TIC para atender a la diversidad. Revista de currículum y formación del profesorado, 16(1), 278-293.

Gillies, R., y Boyle, M. (2010). Teachers' reflections on cooperative learning: Issues of implementation. Teaching and Teacher Education, 26(4), 933-940. DOI: 10.1016/j.tate.2009.10.034

Gómez, I. M., y Ruíz, M. (2018). Interdisciplinariedad y TIC: nuevas metodologias docentes aplicadas a la enseñanza superior. Revista de Medios y Educación, 52, 67-80.

Hammond, J. A., Bithell, C. P., y Jones, L. (2010). A first year experience of student-directed peer-assisted learning. Active Learning in Higher Education, 11(3), 201-212. DOI: $10.1177 / 1469787410379683$ 
Hargreaves, A., y Fullan, M. (2014). Capital profesional. Transformar la enseñanza en cada escuela. Madrid, España: Morata.

Heredia, H., Romero, M., y Amar, V. (2018). Facebook, un espacio para compartir la lectura. Una experiencia en un aula de Educación Secundaria Obligatoria en España. Educacao em Foco, 23(1), 201-224.

Ibarra-Sáiz, M. S., y Rodríguez-Gómez, G. (2011). Aprendizaje autónomo y trabajo en equipo: reflexiones desde la competencia percibida por los estudiantes universitarios. Revista Electrónica Interuniversitaria de Formación del Profesorado, 14(4), 73-85.

Johnson, R. B., y Onwuegbuzie, A. J. (2004). Mixed methods research: A research paradigm whose time has come. Educational researcher, 33(7), 14-26.

Korthagen, F. (2011). Making Teachinig Education Relevant for practice: The pedagogy of realistic teacher education. Orbs scholae, 5(2), 31-50.

Korthagen, F. (2014). In search of the essence of a good teacher: Towards a more holistic approach in teacher education. Teaching and Teacher Education, 20(1), 77-97.

León de Mora, C. (2010). Entornos colaborativos en docencia virtual: redes sociales y wikis. Sevilla: Universidad de Sevilla, Secretariado de publicaciones.

López, C. (2019). La red social Twitter como herramienta didáctica: una metodología de innovación docente en Educación superior. En R. Roig-Vila (Ed.), Innovación e investigación en la enseñanza Superior: Nuevos contextos, nuevas ideas. España: Octaedro.

López Melero, M. (2014). Sin distancias, la cultura escolar se construye. Cuadernos de pedagogía, 447, 84-87.

López Melero, M. (2018). Fundamentos y Prácticas Inclusivas en el Proyecto Roma. Madrid, España: Morata.

Marín, V., y Cabero, J. (2019). Las redes sociales en educación: desde la innovación a la investigación educativa. Revista Iberoamericana de Educación a Distancia, 22(2), 25-33.

Marsh, H. W. (1982). SEEQ: A reliable, valid, and useful instrument for collecting students' evaluations of university teaching. British 
Journal of Educational Psychology, 52(1), 77-95.

Marsh, H. W. (1987). Students' evaluations of university teaching: Research findings, methodological issues, and directions for future research. International Journal of Educational Research, $11(3), 253-388$.

Marsh, H. W. (1991). Multidimensional students' evaluations of teaching effectiveness: A test of alternative higher-order structures. Journal of Educational Psychology, 83(2), 285-296.

Martínez-Clares, P., y González-Morgado, N. (2019). El dominio de competencias transversales en Educación Superior en diferentes contextos formativos. Educacao e Pesquisa, 45, 1-22.

Melife, K., Tigchelaar, A., y Korthanger, F. (2010). Aprender de la práctica. En O. Esteve, K. Melief, y A. Alsina (Eds.), Creando mi profesión. Barcelona, España: Octaedro.

Moriña Diez, A., Perera Rodríguez, V. H., y Melero Aguilar, N. (2014). Análisis de las barreras y ayudas que los estudiantes con discapacidad identifican en la Universidad. Alicante: 3Ciencias.

Núñez Moscoso, J. (2017). Los métodos mixtos en la investigación en educación: Hacia un uso reflexivo. Cuadernos de Pesquisa, 164(47), 632-649.

Odetti, C., y Valentiluz, S. (2017). Jóvenes y Educación. Acerca de las relaciones con las Tecnologías de la Información y la Comunicación en la Escuela Secundaria. Revista de la Escuela de Ciencias de la Educación, 1(12), 133-149.

Okuda Benavides, M., y Gómez-Restrepo, C. (2005). Métodos en investigación cualitativa: triangulación. Revista Colombiana de Psiquiatría, 1(34), 118-124.

Pinos Castillo, L. F. (2018). Análisis comparativo sobre alternativas para sistemas de videoconferencias interactivas en Internet: Caso de estudio Universidad Católica de Cuenca. Revista de Producción Ciencias e Investigación. Pro-Sciences, 8(2), 26-31.

Pozo-Muñoz, C., y Bretones-Nieto, B. (2015). Dificultades y retos en la implantación de los títulos de grado en las universidades españolas. Revista de Educación, 367, 147-172.

Rodríguez, M., López. A., y Martín, I. (2017), Percepciones de los 